\title{
La Facultad de Educación
}

\section{Febrero 1991- Febrero 1995}

Inicia este periodo con el reto de la organización y realización del VIII Encuentro de Facultades de Educación del suroccidente colombiano, realizado entre el 29 y el 31 de marzo de 1991; al cual asistieron cerca de cien representantes de Facultades de Educación y de Instituciones formadoras de maestros, quienes elogiaron la buena organización y la seriedad de sus conclusiones, reconociendo el cálido afecto y la esmerada atención recibida durante su permanencia en la Universidad.

La Facultad de Educación orientó la elaboración de un Plan de Desarrollo, a partir de un proceso participativo y que tenía como fin propiciar propuestas curriculares y un modelo pedagógico que respondiera a las exigencias actuales de la realidad regional y nacional; presentändose en esos momentos los primeros proyectos de reformas a las Facultades de Educación; proyectos liderados por el ICFES, como también la conformación de las primeras comisiones institucionales con el fin de trabajar una propuesta de reestructuración académico-administrativa de la Universidad Surcolombiana; estos principios de cambios a nivel nacional e institucional, llevó a la Facultad a detenerse en su plan de desarrollo, a la espera de las directrices trazadas, tanto a nivel nacional como institucional.

Conscientes de la necesidad de impulsar proyectos de investigación, extensión y docencia; objetivos principales de la Universidad; independiente de la reestructuración académico administrativa que se adoptara; la Decanatura consideró la necesidad de crear cuatro Comités de apoyo con asignación temporal para el cumplimiento de sus compromisos.

EL COMITÉ DE INVESTIGACIÓN logró la sistematización de los procesos de investigación que se desarrollaron en la Facultad a partir de los R.A.E. que será base para definir las políticas investigativas con las políticas universitarias emanadas del CIDEC.

Se promovió la discusión, en la modalidad de seminario taller, que determinó las bases para el replanteamiento del área de investigación de la Facultad y la Universidad en general.

EL COMITÉ DE EXTENSIÓN teniendo en cuenta su acción en el medio como factor de compromiso de la Universidad Surcolombiana con la comunidad se trabajaron propuestas que llevaron a definir acciones concretas de capacitación y prestación de servicios a la comunidad; las cuales podemos resaltar la continuidad del proyecto de Alfabetización; la extensión en Inglés actualmente con una cobertura de 205 estudiantes los cursos de Informática ofrecidos a la comunidad a través del Laboratorio de Informática; los proyectos de capacitación aprobados por el M.E.N.; como el presentado por la profesora Magdalena Arias Bonilla y Angela Rivera: Hacia el desarrollo de la Lengua Materna desde el aula de clase y el proyecto de "Capacitación de Maestros del Huila" elaborado y presentado por los profesores Alba Luz Quintero y Jesús María Vidal, como para mencionar los más reciente.

COMITÉ DE PRACTICA DOCENTE. Se realizó el análisis preliminar para la creación de un Instituto de Aplicaciones Pedagógicas en convenio con la Alcaldía : 
la Secretaría de Educación. El proyecto se encuentra en la etapa de definición con el Centro Docente Agustín Codazzi. Por otro lado, se ajustaron los reglamentos de práctica a las necesidades de la Ley General de Educación y a la modernización del trabajo docente.

COMITÉ

INSTITUCIONAL.
DE grupo interdisciplinario atendiendo a la necesidad de responder al proceso de reestructuración que adelanta la Universidad, para adecuarse a las nuevas exigencias de orden socio.-histórico y ajustarse a la Ley 30 de la Educación Superior y definir criterios para la capacitación de los docentes de la Facultad de la Universidad.

Si bien, no se ha logrado concretar un Plan de Desarrollo para la Facultad, los avances parciales han sido significativos para la definición de la misión, propósitos y objetivos para la Facultad y básicos para las discusiones en los programas.

La administración de la Facultad ha sido consciente de que con el concurso de todos sus estamentos han contribuido en su propio proceso de modernización académico administrativa en el de la Universidad Surcolombiana, aún cuando hay conciencia de que falta mucho por hacer.

Durante este período la Decanatura adelantó una Gestión Administrativa, antes entes de orden institucional, departamental y nacional; destacando como los más importantes los siguientes:

1. El apoyo económico de las administraciones anteriores de la Universidad, para acondicionar las instalaciones y mejorar el ambiente físico de trabajo.

2. Se revivió la edición de la revista de la Facultad PAIDEIA.

3. Avanzar en la implementación de la Informätica en la docencia requerida por la mayoría de los Programas con el apoyo económico representativo del señor Contralor del momento Doctor Jaime Losada, que fue complementado por el
ICFES y la Universidad para adquirir los computadores y crear el LABORATORIO DE INFORMÁTICA de la Facultad. Desde entonces se han programado permanentemente cursos para profesores y se estudia la forma de prestar servicios al personal de la Universidad y fuera de ella.

4. En el campo académico se gestionó ante el ICFES la licencia de funcionamiento del Programa de Lenguas Modernas negada en tres ocasiones anteriores, la aproba- ción de la Especialización en Comunicación y Creatividad para la docencia; la puesta en funcionamiento de la Especialización para la Enseñanza de la Biología, en convenio con la Universidad del Tolima y la Maestría en Educación y Desarrollo Comunitario en convenio con el CINDE, Programas que se encuentran trabajando todos con excelente ritmo y calidad académica.

5. Se gestionó ante el ICFES la creación de la biblioteca especializada para los Postgrados, y se obtuvo un aporte de 4 millones.

6. La financiación del ICFES para traer profesionales calificados a nivel nacional e internacional para adelantar seminarios y conferencias complementarias para la especialización en Comunicación y Creatividad para la docencia.

Respondiendo al propósito de dar mayor autonomía y responsabilidad a los Programas se descentralizaron los tramites para que ellos mismos realicen los estudios necesarios, elaboren proyectos alternativos de profesionalización, capacitación y servicios a la comunidad en concordancia con las nuevas disposiciones. Los proyectos de trabajo por Programa fueron dados a conocer en el Consejo Académico y dieron origen a la adopción a una nueva modalidad de soporte para la asignación de carga académica.

Por iniciativa del profesor Nicolás Núñez, la Decanatura adelantó conversaciones para elaborar y presentar un proyecto macro para la creación y puesta en funcionamiento de un CENTRO REGIONAL PARA LA ENSEÑANZA DE LA INFORMÁTICA 
C.R.E.I. Este proyecto fue traducido al inglés por el Programa de Lenguas Modernas para presentarlo ante el C.R.E.I. Regional que funciona como una dependencia del DANE, e igualmente se presentó ante Planeación Nacional y COLCIENCIAS por intermedio del ICFES. El proyecto ha tenido conceptos favorables en cada una de las instancias como consta en la carta del Director General de Procesamiento de Datos quien nos comunica que obtuvo información y calificación por parte del Programa intergubernamental. de INFORMÁTICA, por lo cual estimamos que muy pronto estará debidamente aprobado y financiado.

Presentamos ante el ICFES un proyecto institucional por SETENTA Y CINCO MILLONES DE PESOS $(\$ 75.000 .000)$ para mejorar los laboratorios de física e implementar la informática en cada uno de los programas de la Facultad, para la adquisición de equipos audiovisuales para los Programas. El proyecto fue calificado por la comisión técnica del ICFES y la Junta Directiva con base en la disponibilidad presupuestal del momento nos asignó veinticinco millones $(\$ 25.000 .000)$, pero desafortuna-damente por cambios de dirección en el ICFES, este proyecto tuvo cambios de designación a última hora y a la Facultad de Educación le correspondió únicamente 10 millones de pesos que se invirtieron en los Laboratorios de Física; el resto pasó a la Facultad de Ingeniería.

Nuestro esfuerzo para dinamizar el tramite de los requeri-mientos docenteadministrativos de responsabilidad de la Decanatura con seguimiento diario de los procesos y un inventario semanal de las acciones y conceptos.

Contamos con la colaboración de profesores idóneos para adelantar los estudios y propuestas solicitados. A ellos agradecemos su permanente disposición para colaborarnos, para facilitar la orientación de los trabajos, pues a pesar de que estan sobrecargados académicamente, la Facultad siempre tuvo la posibilidad de responder con oportunidad y eficiencia a cada requerimiento de las directivas de la Universidad y de cada una de las instituciones nacionales e internacionales con las que nos ha correspondido relacionarnos. De ello quiero resaltar los procesos de des-centralización, de reestructuración de varios programas y Especializaciones dando tramite unos hasta el Consejo Superior y otros están en el Consejo de Facultad.

Los Programas de Pregrado y Especializaciones que han cumplido sus tramites e inician labores en 1995 son:

a. Se continuó y finalizó el proceso de reestructuración del Programa de Lingüística y Literatura que se convirtió en la Licenciatura de Español y Comunicación Educativa; ésta fue aprobada en los Consejos de Facultad, Académico y Superior Universitario.

b. Se presentó ante los Consejos Académico y Superior el proyecto C.I.P.C.I.T. (CENTRO DE INTERACCIÓN DEL PUBLICO CON LA CIENCIA Y LA TECNOLOGÍA), el cual culminó con su aprobación.

c. Se presentó estudio de factibilidad, elaboró, estudió y ajustó el proyecto de Especialización en Fisica, aprobado en Consejo de Facultad, Académico y Superior. d. Elaboración de dos proyectos del área de matemáticas: Uno de Especialización en Matemática y otro para la creación de una Licenciatura en Matemáticas modalidad semipresencial; proyectos que pasan al Consejo Académico.

e. En el semestre B-1994 se inició y culminó con éxito el desarrollo del primer semestre de Licenciatura en Educación Física en la ciudad de Florencia, en convenio con la Universidad de la Amazonía.

f. Durante el semestre B-1994 se presentó y aprobó el proyecto para sacar una promoción de Licenciatura en Educación Física en la ciudad de Pitalito, proyecto que se implementara en este semestre. 
g. Semestre b-94 se elaboró y aprobó la realización de un postgrado a nivel de Especialización en docencia de la Educación Física en la sede principal de la USCO. Actual-mente están abiertas las inscripciones y se aspira iniciar cla-ses el 24 de febrero de 1995.

h. Propuesta de homologación de la Licenciatura en Preescolar del Instituto Técnico Superior de San Andrés Isla, la cual fue enviada.

i. Propuesta de nivelación en Preescolar para estudiantes del Instituto Tolimense de Formación Técnica Profesional, según convenio entre las dos instituciones, propuesta que se implementara en este semestre.

j. El Programa de Preescolar culminó su reestructuración, cumplió con el tramite ante los Consejos de Facultad, Académico y Superior, 10 que dio para convertir el Programa de Preescolar en Educación Infantil.

k. Propuesta para el diseño, desarrollo y evaluación de un Programa de inducción en docencia. Responsable: Aura Helena Bernal de Rojas.

1. Propuesta para la creación del postgrado en Desarrollo Humano y Educación Sexual. Responsables: Margarita Chacón y Nicolás Núñez; aprobado por todas las instancias y se da inicio en el primer semestre de 1995.

m. En el semestre B-94 se culminó y aprobó por todas las instancias la Especialización en Planeación del Desarrollo Educativo y Cultural. quienes iniciaron clases en el primer semestre de 1995.

Nace de la Facultad de Educación, en cabeza del profesor William Fernando Torres el pregrado en Comunicación Social y procesos culturales con énfasis en radio y T.V.; cumpliendo todos los tramites e iniciando labores en el semestre A de 1995. Agradecemos a cada una de las personas que intervinieron en nuestra administración, a cada uno de los directivos de la Universidad que comprendieron y apoyaron cada una de iniciativas no habriamos podido trabajar. 